\title{
Tire Dynamics Deflection Assessment from IMU Measurement
}

\author{
[Pornporm Boonporm and Narawith Phitchart]
}

\begin{abstract}
One important objective of vehicle suspension design is Safety, considering road holding performance in terms of its cornering, braking and traction. In this sense, we are interested in studying the tire deflection since it relates to how good the road holding is via the variation of normal tire load. We apply the full car suspension model with some measured or estimated parameters as reference model. Vehicle body's accelerations and angular rates are measured. The sprung mass displacement, body roll and pitch angle can be calculated and compared to the measurement values. The errors and error gradients are derived and then adjusted via gain update estimation consulting the fuzzy logic rules in order to corrected parameter. Finally suspension deflection and tire deflection are predicted via our algorithm. The results are in accordance with the physical meaning and subjective assessment by experienced driver.
\end{abstract}

Keywords - vehicle kinematics; full car suspension model; tire deflection; parameters tuning.

\section{Introduction}

Safety is one of the main criteria for designing vehicle suspension, since most of accidents come from rollover and yaw stability. In order to keep good road holding performance, it depends on the dynamics interaction between tire and ground, defined in term of tire deflection. This idea comes from the lateral and longitudinal forced generated by tires are proportional to vertical tire load during driving. Considering a tire-generated normal force that responses like a spring, it can be directly related to tire deflection. The road holding performance of a suspension can therefore be quantified in terms of the tire deflection performance.

There are some former scientific works dealt with tire deflection analysis and related topic in [1] proposed a model of interaction between the suspension dynamics and the tireroad forces, on the basis an analysis focused on the evaluation metrics for handling performances based on optimal predictive control with road preview. The algorithm to estimate tire vertical forces presented by [2] applied Roll and combined

Pornporm Boonporm

School of Mechanical Engineering, Suranareee University of Technology, Thailand.

Narawith Phitchart

School of Mechanical Engineering, Suranareee University of Technology, Thailand. longitudinal-lateral dynamics. The proposed method uses measurements from currently available sensors like accelerometers and relative suspension sensors. There is some work about design and simulation of a variable stiffness and damping coefficient stated in [3]. Numerical simulation has been performed in MATLAB Simscape and experimentations have been performed to validate the theoretical model. Quarter car simulation has been used to quantify comfort and handling evaluation. One of the applications demonstrated in [4]. They tried to estimate the tire vertical deflection in a motorcycle suspension that was assumed to be directly related with the contact forces between the tire and the road. Their work was based on the Kalman-filtering techniques. The kinematics data measurement was one of the useful choices for vehicle handling as written in [5].

There was some study about the tire vertical dynamics for friction coefficient, tire behavior and etc. as in [6]. The parameter tuning methods and algorithms via fuzzy logic were interested. In [7] defined PI fuzzy logic self-tuning via gradient descent method. The application in industrial process was demonstrated in [8] by also applying the self-tuning fuzzy logic. Matlab /Simulink was the useful tool for system determination, control and validation as stated in [9]

Our study aims at providing a handling performance evaluation by means of tire deflection approximation. We focus on road vehicles model with full car suspension model. The kinematics measurement data acquired by inertial measurement unit integrated with GNSS are used for our analysis. Problems arise because we do not know the exact parameter values, then they are estimated or observed and compare to the measurements. These are updated via parameter tuning method. The 7 DOF model is written in state space form for $14 \times 14$ system matrix and 4 inputs from road profiles at each wheel, in propose of deriving transfer function for interested pair of inputs and outputs, as well as Simulink block diagram which is useful in the simulation and system analysis. .

\section{System Installation}

The vehicle, sensor and procedures are identified and modeled as in following subsection

\section{A. Tested Vehicle}

Passenger car equipped with IMU as shown in fig. 1 is used. The vehicle specification in overview is described in table 1 . 
Proc. of the Sixth Intl. Conf. Advances in Civil, Structural and Mechanical Engineering - CSM 2018

Copyright (C) Institute of Research Engineers and Doctors, USA. All rights reserved.

ISBN: 978-1-63248-150-4 doi: 10.15224/978-1-63248-150-4-33

Front suspension is McPherson strut and the rear one is torsion beam.

TABLE I. BASIC SPECIFICATIONS OF TESTING VEHICLE.

\begin{tabular}{|c|c|}
\hline Model & Toyota Vios 2008 (NCP-93) \\
\hline Engine & 1NZ-FE 1.5L \\
\hline Transmission & 4-Speed AT \\
\hline Wheelbase $(\mathbf{m m})$. & 2550 \\
\hline Length $(\mathbf{m m})$. & 4300 \\
\hline Width $(\mathbf{m m})$. & 1700 \\
\hline Height $(\mathbf{m m})$. & 1460 \\
\hline Weight $(\mathbf{K g})$. & $1045-1060$ \\
\hline
\end{tabular}

The static weight distribution of the vehicle is measured for each axle and wheel form the load cell and the values are shown in table 2 .

TABLE II. STATIC WEIGHT DISTRIBUTION.

\begin{tabular}{|c|c|c|}
\hline & Front & Rear \\
\hline Left (Kg.) & 330 & 205 \\
\hline Right (Kg.) & 325 & 210 \\
\hline
\end{tabular}

\section{B. Sensor Feature and Mounting}

Inertial measurement unit integrated with GNSS is the only a sensor for our measuring the vehicle's kinematics values such as acceleration in three axis, rotation angle and rate. Its characteristics are written in table 3.and 4 for IMU and GNSS respectively. Demonstrating in figure 1 , sensor is mounted at the near from the CG with the offset distance
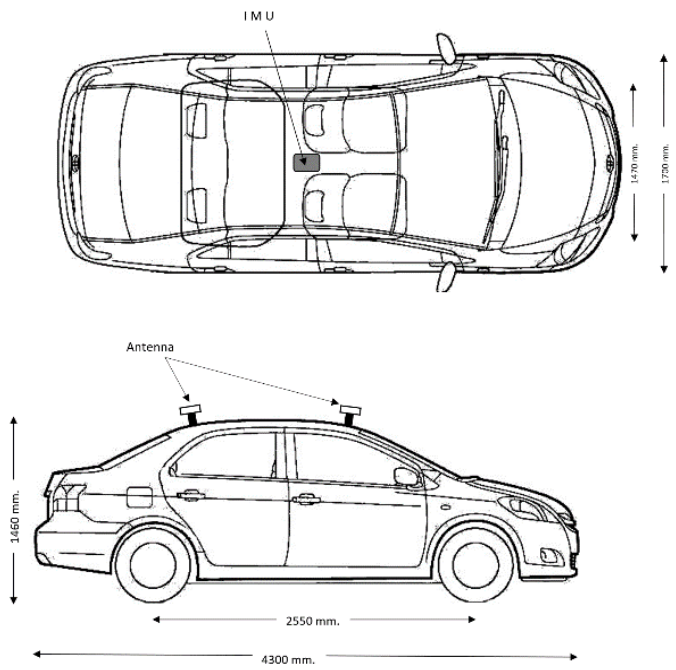

Figure 1. Test vehicle and sensor mounting.

TABLE III. IMU SPECIFICATIONS.

\begin{tabular}{|c|c|c|}
\hline Parameter & Accelerometer & Gyroscopes \\
\hline Range (dynamic) & $4 \mathrm{~g}$ & $500 \% \mathrm{~s}$ \\
\hline Noise density & $150 \mu \mathrm{g} \sqrt{\mathrm{Hz}}$ & $0.009^{\circ} / \mathrm{s} \sqrt{\mathrm{Hz}}$ \\
\hline
\end{tabular}

\begin{tabular}{|c|c|c|}
\hline Cross-axis alignment error & $<0.05^{\circ}$ & $<0.05^{\circ}$ \\
\hline Bandwidth & $400 \mathrm{~Hz}$ & $400 \mathrm{~Hz}$ \\
\hline
\end{tabular}

TABLE IV. GNSS SPECIFICATIONS.

\begin{tabular}{|c|c|}
\hline Parameter & Value \\
\hline Supported navigation systems & GPS L1, L2, L5 \\
\hline Update rate & $20 \mathrm{~Hz}$ \\
\hline Horizontal position accuracy & $1.2 \mathrm{~m}$ \\
\hline Velocity accuracy & $0.01 \mathrm{~m} / \mathrm{s}$ \\
\hline
\end{tabular}

\section{Full Car Suspension Model}

The dynamic model of a 7-DOF full car system is chosen as depicted in figure 2 with sets of differential equations in state space form as in equation (1). The system consists of 7 dynamics equations from bouncing, pitching, rolling of the sprung mass mode, and four equations of unsprung mass in vertical direction. Road profiles are inputs at each wheel. A and $\mathrm{B}$ is system matrix and input matrix respectively, which can be derived from 7 equation of motion as state above.

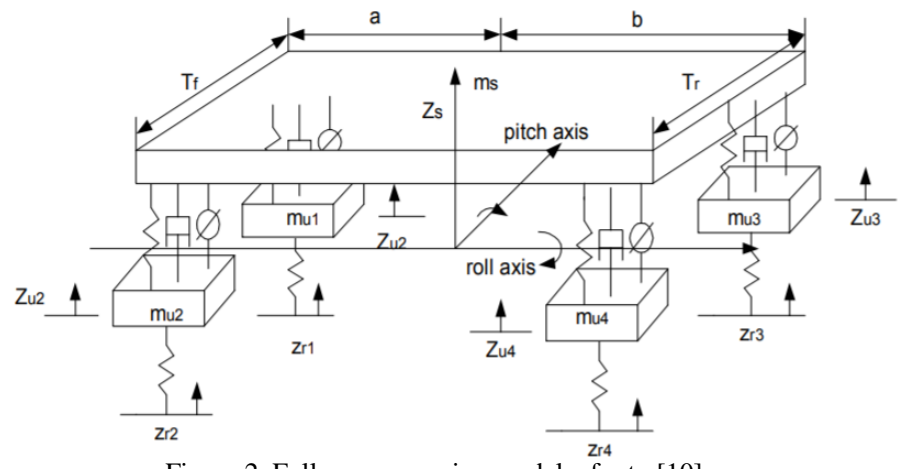

Figure 2. Full car suspension model refer to [10]

$$
\dot{x}=A x+B z_{r}
$$

Due to the tedious work for calculating, we do simulation using math model that can be apply in Matlab/Simulink model for full car as demonstrate in figure 3.

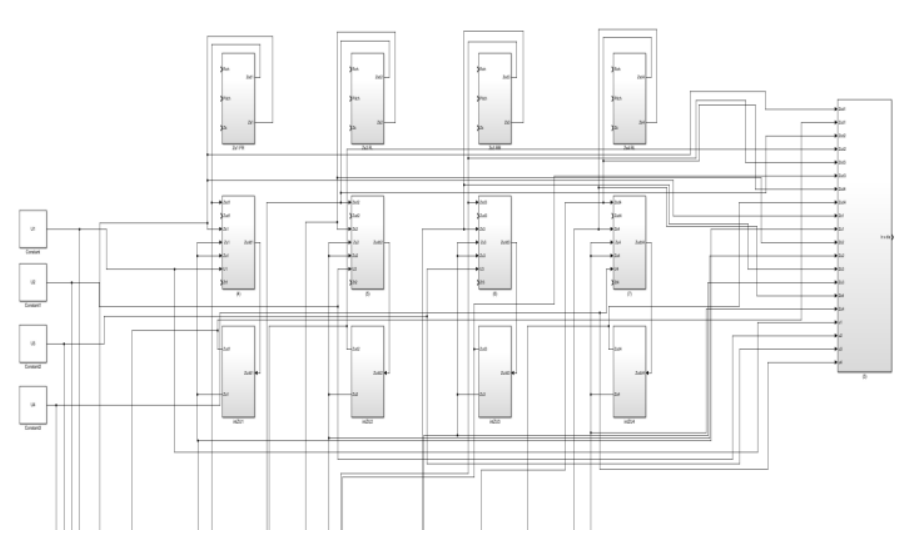

Figure 3. Full car Matlab/Simulink model. 


\section{Preliminary Estimation}

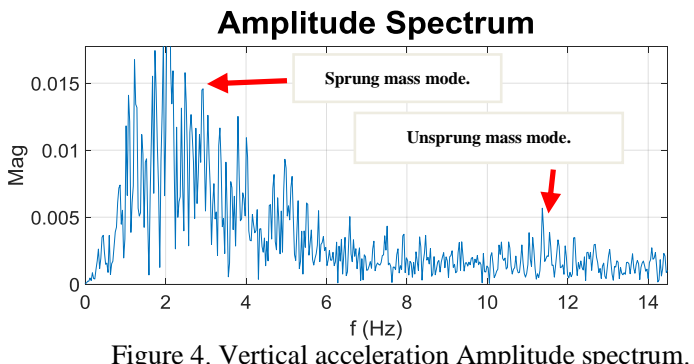

The suspension parameters are defined as front and rear spring stiffness, front and rear damping coefficient, tire stiffness and unsprung mass at each wheel are approximated as the characteristic of the response. We investigated spectrum analysis especially in vertical direction like displacement, velocity and acceleration depending on the frequencies, as well as roll rate and pitch rate in any cases from the experiment data as one example in figure 4 . We tuned and at the same time compare to frequency response analysis in order to evaluate the natural frequency of sprung and unsprung mass mode in vertical direction. For sprung mass natural frequencies, they vary between $1.7-2.1 \mathrm{~Hz}$. It is a little bit difference among the wheels, while the unsprung mass mode are approximate as $11-12 \mathrm{~Hz}$ depended on tire pressure. From the second mode vibration analysis in frequency response, tire stiffness is calculated.

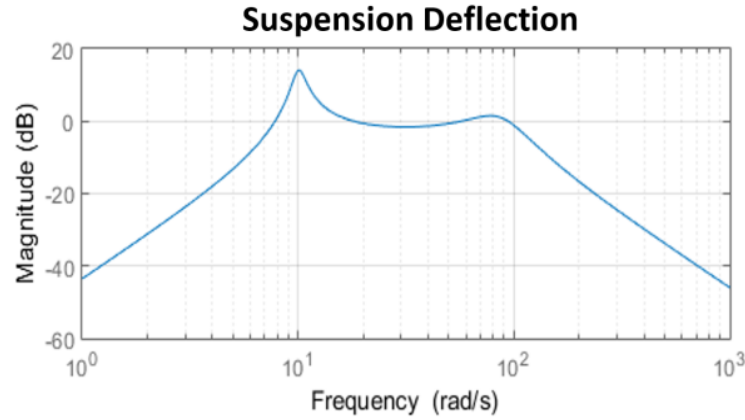

Figure 5. Example of frequency response of suspension deflection for front left wheel as reduced 2 DOF model.

Tire Deflection

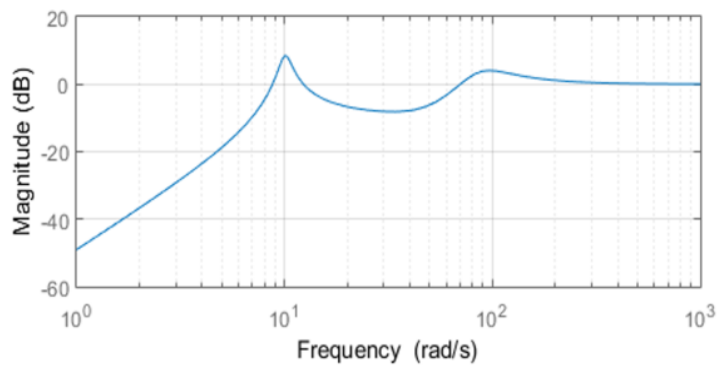

Figure 6. Example of frequency response of tire deflection for front left wheel as reduced $2 \mathrm{DOF}$ model.

Figure 5 and 6 show approximate frequency response of the preliminary estimated parameter as suspension deflection or so called rattle space and tire deflection which is related to the handling.

\section{Tire Deflection Estimation}

Since the accelerations and angular rates are received from IMU, we need to approximate via the experiment data as stated in figure 7. Road profile input are generated refer to the real situation as step input and half sine curve as a road bump. They are the same inputs for left and right wheel but the time delay is included as input for rear wheel depended on the vehicle speed. Three values like bounce, roll and pitch are calculated form the model and compare to the measurement with the signal preprocess such as filtering and smoothing from similar input cases. Unfortunately, some error occurs because of the parameter uncertainty form the primary estimation. From the model, we need to update sprung mass, wheels mass, front spring stiffness, rear spring stiffness, front damper, rear damper and tire stiffness. All masses are 10\% bounded but the stiffness and damping coefficient are defined as $20 \%$ subjected to their nonlinearities.

The error gradient is determined via the partial derivative respect to bounce, roll and pitch. From these gradients, the direction maximum rate of change is evaluated and sort for direction change. From the data, we can process the most to the least important parameter update. The fuzzy self-tuning for each variable are separately determined. The error level in linguistic level are divided into 7 levels as negative big (NB), negative(N), negative small(NS), Zero(Z), positive small(PS), positive $(\mathrm{P})$ and positive big( $\mathrm{PB})$; while the change of error are defined only in three levels as negative $(\mathrm{N})$, zero( $\mathrm{Z})$ and positive(P). The outputs are parameter update values. The iterations are done until error converge to zero.

The tuned parameters is obtained, so that from state space model, the approximate transfer function can be derived via matlab programming. Finally the tire deflection from each wheel is calculated.

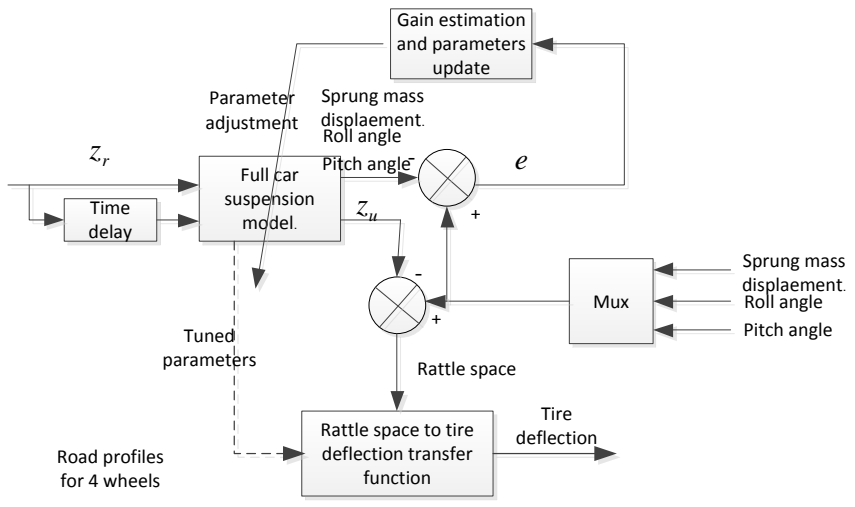

Figure 7.Model and estimation method.

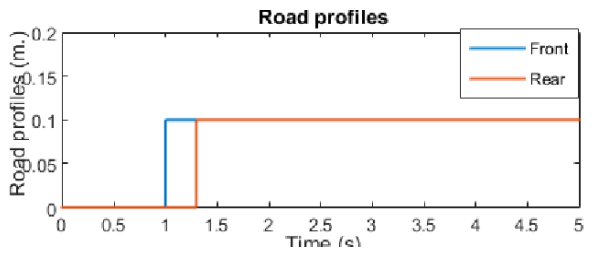

Figure 8.Model and estimation method. 
For parameter tuning purpose, we tested in step environment which the inputs are as road profile in figure 8 . We assumed the same input in both front wheels and the time delay are added for rear wheels. The time gap depended on the vehicle speed.
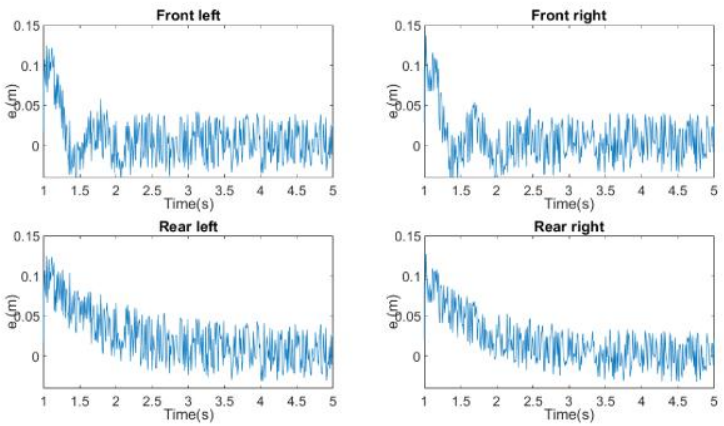

Figure 9. Sprung mass displacement error.

Although there was one unsprung mass displacement measured at CG, we can transform to the movement at the position of each ball joint as express in following equations (2)-(5)

$$
\begin{gathered}
z_{F L}=z+l_{f} \theta-b_{L} \varphi \\
z_{F R}=z+l_{f} \theta+b_{R} \varphi \\
z_{R L}=z-l_{f} \theta-b_{L} \varphi \\
z_{R R}=z-l_{f} \theta+b_{L} \varphi
\end{gathered}
$$

The error at each ball joint was investigated in figure 9 and the parameters update as spring stiffness and damping coefficient are demonstrated in figure 10 and 11 respectively. The initial guest of the spring stiffness was $50000 \mathrm{~N} / \mathrm{m}$, that received from manufacture. After 40 iterations it reached the steady state which is $50040 \mathrm{~N} / \mathrm{m}$. The damping coefficient start from $1200 \mathrm{Ns} / \mathrm{m}$ and became steady when 60 iterations passed.

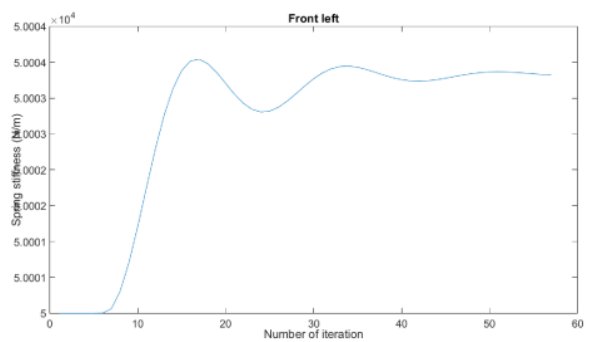

Figure 10. Spring stiffness tuning.

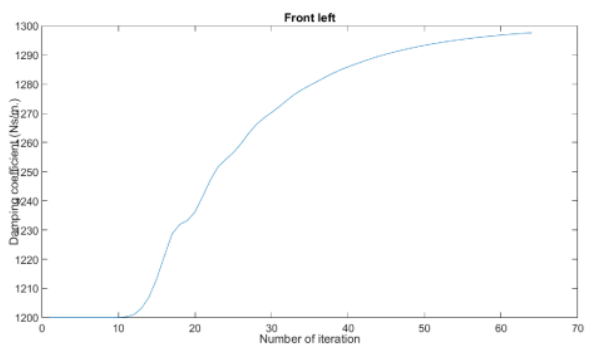

Figure 11. Damping coefficient tuning.

\section{Experiment results}

The showcase in this paper, we chose the driving curve as in figure 12 in clockwise cornering as the driving information in figure 13 and 16. The longitudinal and lateral acceleration are depicted for evaluating the driving situation. Roll angle and pitch angle are arising due to the vehicle suspension and the road profile like tilt, bank, hole and etc. The suspension deflection of each wheel is shown in figure 14 and 17 as well as the tire deflection in figure 15 and 18 . Semi-active suspension is used in our experiments. The damping coefficient can be adjust in 30 levels. The example case F0R0 mean the damping level is in level 0 in both front and rear, and the same did the level 18.

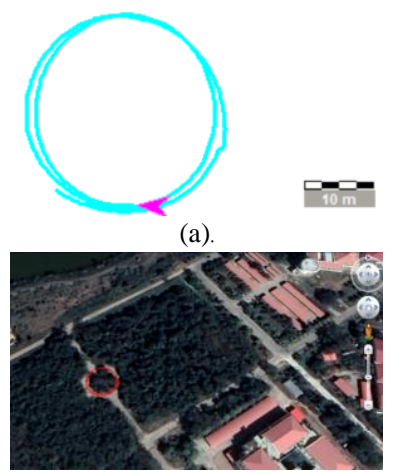

Figure 12 (a) Test path, (b). Test path in real environment.
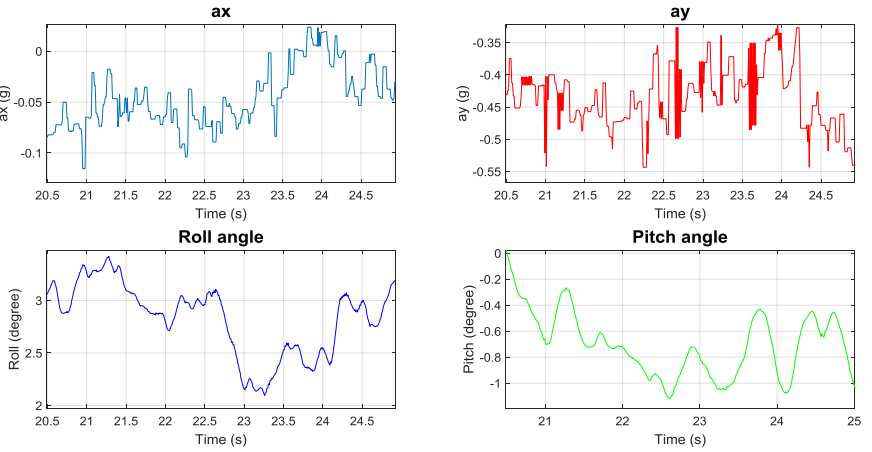

Figure 13.Measured data damp level F0-R0.
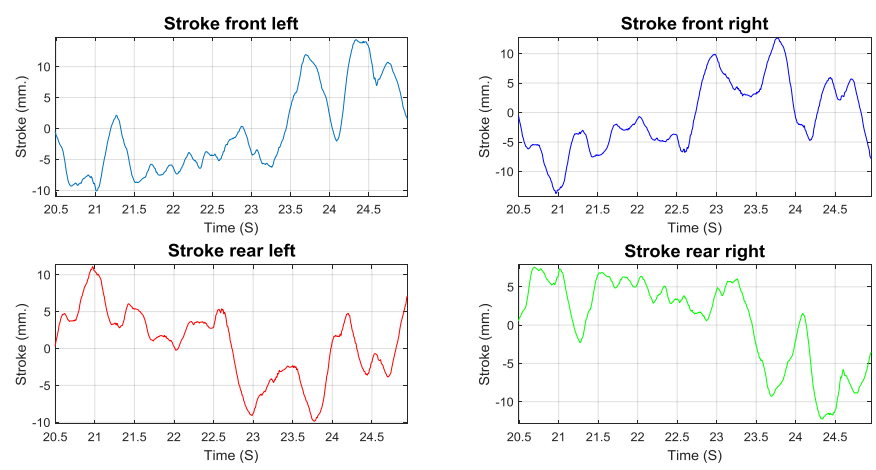

Figure 14.Suspension deflection damp level F0-R0.

When the vehicle is under a longitudinal acceleration from starting, the rear suspension displacements are compressed at 
the same time the front are rebound. While cornering, lateral acceleration induces a roll angle. In both cases referring from suspensions stroke depicted in figure 9 and 11, the right side of vehicle is deflected more than the left one because of weight transfer from counter-clockwise cornering, and they act in the same trend. One example shown, at the time between $22.5 \mathrm{sec}$. to $24 \mathrm{sec}$., the effect from damp level 0 bump and rebound rate are slower than damp level 18 because it has more compression than the other.

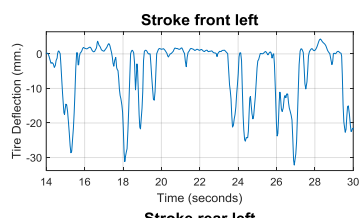
Stroke rear left

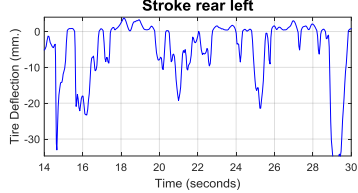

Figure 15. Tire deflection damp level F0-R0.
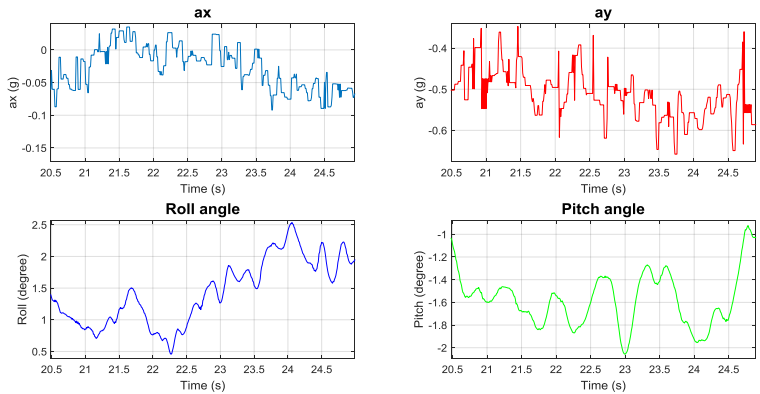

Figure 16.Measured data damp level F18-R18.

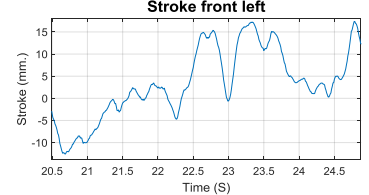

Stroke rear left

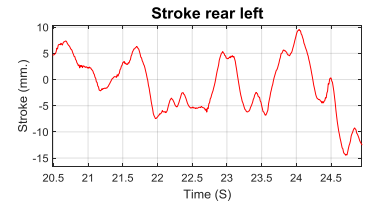

Figure 17.Suspension deflection damp level F18-R18
The last step, tire deflections are approximate and the results are in an agreement with our subjective evaluation via the questionnaire from experienced driver.

The tire deflection of both cases are not significantly different from both cases of shock absorber but damp level 18 has more displacement on tire than damp level 0. Objective validation is difficult to conclude, since it depends upon many factors like a tire configuration, suspension arrangement and etc., although there are many studies available. The result is evaluated via subjective assessment and it agrees with the vehicle kinematics information.
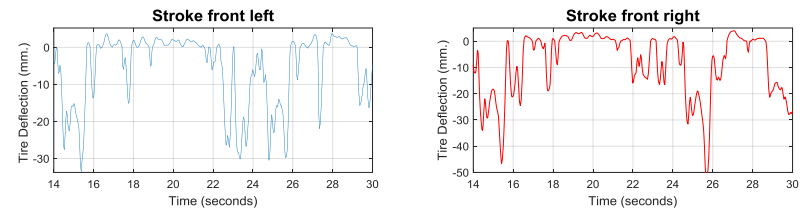

Stroke rear left
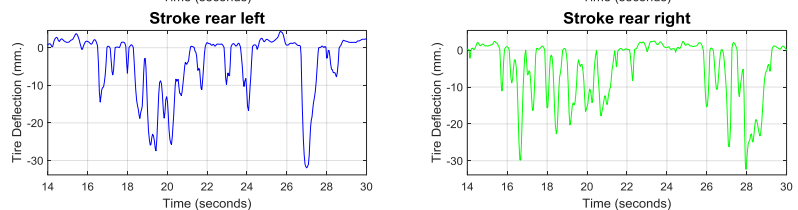

Fig 13. Tire deflection damp level F18-R18.

\section{v. Conclusion}

This study focused on calculation of tire deflection, which is first step for objective evaluation of vehicle handling. Unfortunately, we do not get all important data so that the estimation process and tuned parameters help us to calculate the tire deflection value. The gain updated variables are calculated via the model compare to the experiment data assisted with the parameter tuning. Finally the tire deflections are derived from this algorithm.

\section{References}

[1] C. Spelta, D. Delvecchio, R. Cantoni, R. Lazzari, and S. M. Savaresi, "Analysis and Design of Handling-Oriented Control Strategies for SemiActive Suspensions," Joint 48th IEEE Conference on Decision and Control and 28th Chinese Control Conference, Shanghai, P.R. China, December 16-18, 2009.

[2] M. Doumiati, A. Victorino, A. Charara, G. Baffet, D. Lechner, "An estimation process for vehicle wheel-ground contact normal forces," Proceedings of the 17th World Congress The International Federation of Automatic Control Seoul, Korea, July 6-11, 2008.

[3] L. M. Jugulkar, S. Singh, and S. M. Sawant, " Analysis of suspension with variable stiffness and damping force for automotive applications", Advance in Mechanical Engineering 2016, Vol.8(5) 1-19

[4] D. Delvecchio, C. Spelta, and S. M. Savaresi, "Estimation of the Tire Vetical Deflection in a Motorcycle Suspension via Kalman-Filtering Techniques, " IEEE International Conference on Control Applications (CCA) Part of 2011 IEEE Multi-Conference on Systems and Control Denver, CO, USA. September 28-30,2011.

[5] E. Prompakdee, P. Boonporm, and S. Rooppakhun, "The Influence of Weight Distribution on the Handling Characteristics of Inter city Bus under Steady State Vehicle Cornering Condition,": 5th International Conference on Transportation and Traffic Engineering (ICTTE 2016) Volume 81, 2016.

[6] C. Zoller, P. Wagner and H. Winner,"Tires and Vertical Dynamics of Wheeled Mobile Driving Simulator", Transportation Research Part F: Traffic, Psychology and Behaviour, Elsevier, Nov. 2017.

[7] A.H. Habbi and M Zelmat, "Fuzzy Logic Based Gradient Descent Method with Application to a PI Type Fuzzy Controller Tuning: New Results" IEEE International Symposium on Computational Intelligence and Intelligent Informatics, Morocco, 2007.

[8] P.M. Mary and N.S. Marimuthu, "Design of Self-tuning Fuzzy Logic Controller for the Control of an Unknown Process" IET Control Theory and Applications, 2008.

[9] L. Cammarata and L.Yliniemi, "Development of Self-tuning Fuzzy Logic Controler for a Rotary Dryer" Report A. No. 10, Uniersity of Oulu, 1999.

[10] R. Darus, Y.. Sam, "Modeling and Control Active Suspension System for a Full Car model", $5^{\text {th }}$ International Colloquium on Signal Processing and its Applications, 2009. 